\title{
Erratum to: NIV by an interdisciplinary respiratory care team in severe respiratory failure in the emergency department limited to day time hours
}

Christian Michael Horvath $^{1}$ - Martin Hugo Brutsche ${ }^{1}$ Otto Dagobert Schoch ${ }^{1}$. Bernarde Schillig $^{1} \cdot$ Florent Baty $^{1} \cdot$ Dieter vonOw $^{2} \cdot$ Jochen Julius Rüdiger $^{1}$

Published online: 1 November 2016

(c) SIMI 2016

\section{Erratum to: Intern Emerg Med}

DOI 10.1007/s11739-016-1546-z

In the original publication the second author's name was incorrectly published as Martin Horvath Brutsche. The correct name should read as Martin Hugo Brutsche.

The online version of the original article can be found under doi:10.1007/s11739-016-1546-z.

Christian Michael Horvath

christian.horvath@swissonline.ch

1 Pneumology and Sleep Medicine, Kantonsspital St. Gallen, Rorschacherstrasse 95, 9007 St. Gallen, Switzerland

2 Emergency Department, Kantonsspital St. Gallen, Rorschacherstrasse 95, 9007 St. Gallen, Switzerland 